\title{
Accurate classification of 28 objects detected in the 39 months Palermo Swift/BAT hard X-ray catalogue
}

\author{
P. Parisi ${ }^{1,2}$, N. Masetti ${ }^{1}$, E. Jiménez-Bailón ${ }^{3}$, V. Chavushyan ${ }^{4}$, R. Landi ${ }^{1}$, A. Malizia ${ }^{1}$, \\ E. Palazzi ${ }^{1}$, L. Bassani ${ }^{1}$, A. Bazzano ${ }^{2}$, A.J. Bird ${ }^{5}$, G. Galaz ${ }^{6}$, D. Minniti ${ }^{6,7}$, L. Morelli ${ }^{8}$, \\ M. Schiavone ${ }^{1}$ and P. Ubertini ${ }^{2}$ \\ ${ }^{1}$ INAF - IASF di Bologna, Italy. \\ ${ }^{2}$ INAF - IASF di Roma, Italy. \\ ${ }^{3}$ Universidad Autónoma de México, Mexico DF \\ ${ }^{4}$ INAOE, Puebla, Mexico \\ ${ }^{5}$ University of Southampton, UK \\ ${ }^{6}$ Pontificia Universidad Católica, Santiago, Chile \\ ${ }^{7}$ Specola Vaticana, Città del Vaticano \\ ${ }^{8}$ Università di Padova, Italy \\ E-mail: parisi@iasf-roma.inaf.it
}

\begin{abstract}
Through an optical campaign performed at 4 telescopes located in the northern and the southern hemispheres, plus archival data from two on-line sky surveys, we have obtained optical spectroscopy for 28 counterparts of unclassified or poorly studied hard X-ray emitting objects detected with Swift/BAT and listed in the 39 months Palermo Swift/BAT hard X-ray catalogue. We have been able to pinpoint the optical counterpart of these high energy sources by means of X-ray observations taken with Swift/XRT or XMM which allowed us to restrict the positional uncertainty from few arcmin to few arcsec; satellite data also provided information on the X-ray spectra of these objects. We find that 7 sources in our sample are Type 1 AGN while 20 are Type 2 AGN, with their redshifts lying between 0.009 and 0.075 ; the remaining object is a Galactic cataclysmic variable $(\mathrm{CV})$. In this work we provide optical information for all 28 sources and the results of the soft X-ray analysis of 3 out of 5 AGN observed with XMM/Newton.
\end{abstract}

The Extreme and Variable High Energy Sky - extremesky2011,

September 19-23, 2011

Chia Laguna (Cagliari), Italy 


\section{Introduction}

The Swift mission was designed to study cosmic gamma-ray bursts (GRBs) in a multiwavelength context ([7]), but it is also able to perform dedicated X-ray and UV-optical observations as well as surveys of the entire sky. Swift carries three instruments, i.e. the burst alert telescope (BAT; [1]), the X-ray telescope (XRT; [2]) and the ultraviolet/optical telescope (UVOT; [13]) and therefore can detect and follow up X-ray emitting objects over a wide range of wavelengths.

In particular, BAT, the high energy instrument, is a coded mask detector operating with good sensitivity in the energy range 14-195 keV over a field of view of $1.4 \mathrm{sr}$ with a point source location accuracy of $1^{\prime}-4^{\prime}$ ([7]) depending on the source intensity. Its sensitivity is estimated to be $\sim 1$ mCrab at high Galactic latitudes and $\sim 3$ mCrab over the Galactic plane.

This instrument is not only able to detect GRBs, but also to perform highly sensitive hard Xray surveys (e.g. [4], [5], [14]). In particular, the BAT surveys allow the study of the extragalactic X-ray sky, and the observation of many absorbed AGNs which are often missed by lower energy instruments. Quantifying the number of such absorbed objects, especially at low redshifts, is very important if one wants to understand the accretion mechanisms at work in AGNs and to estimate the contribution of all AGN to the cosmic X-ray background ([3]). However, many of the objects listed in the BAT surveys are still unclassified or poorly studied and hence they need optical follow up work to be fully characterized.

For this work we have selected from the 39 months Palermo Swift/BAT AGN survey ([4]), a group of objects (28 in total) either without optical identification, or not well studied or without published optical spectra. Following the method applied by [8] and references therein or [11] for the optical spectroscopic follow up work of unidentified INTEGRAL and/or BAT sources, we determine the nature of these 28 selected objects by means of X-ray observations (to pinpoint the likely X-ray counterpart) and optical measurements (to provide the source classification).

In the following sections we show the results obtained with our optical spectroscopic campaigns and we discuss in detail the results of the X-ray analysis of 3 out of 5 objects observed by $\mathrm{XMM} /$ Newton.

\section{Optical analysis}

The identification of a convincing X-ray counterpart of the BAT source is a fundamental step in order to reduce the positional uncertainty from few arcmin to few arcsec and consequently perform optical follow up work on the likely optical association/s; for this reason we have first analysed a set of X-ray observations performed using Swift/XRT for 23 out of 28 objects, while for the remaining 5 sources archival XMM-Newton data have been considered. The details of the X-ray analysis can be found in [12] where we also describe the optical campaigns we have done, the telescopes used, the data reduction adopted as well as the classification criteria employed.

The optical analysis of the 28 objects in our sample reveals that 27 are AGNs and 1 is a Cataclysmic Variable (CV). Among the sample of extragalactic objects 7 are Type 1 AGN (of which 5 are seyferts of intermediate types 1.2-1.9 and one is a Narrow Line seyfert 1) while 20 are Type 2 AGN (including a few showing LINER type signatures); their redshifts lie between 0.009 and 0.075 , i.e. they are all local AGN. The main results of our optical study are reported in Table 
1 and 2, where we list for each source the $\mathrm{H}_{\alpha}, \mathrm{H}_{\beta}$ and [OIII] fluxes, the classification, the redshift estimated from the narrow lines, the luminosity distance given in $\mathrm{Mpc}$, the Galactic color excess and the color excess local to the AGN host and the name of the optical counterpart in the NED online catalogue ${ }^{1}$.

For the CV, PBC J0826.3-7033, we report the $\mathrm{H}_{\alpha}, \mathrm{H}_{\beta}$ and HeII $\lambda_{4686}$ equivalent widths and fluxes, the $R$ magnitude extracted from the USNO-A2.0 catalogue ([9]), the extinction and the source distance (see Tab. 3).

Table 1: Main results obtained from the analysis of the optical spectra of the 7 type 1 AGN of the present sample of Swift sources.

\begin{tabular}{|c|c|c|c|c|c|c|c|c|c|}
\hline \multirow[t]{2}{*}{ Object } & \multirow[t]{2}{*}{$F_{\mathrm{H}_{\alpha}}{ }^{*}$} & \multirow[t]{2}{*}{$F_{\mathrm{H}_{\beta}}$} & \multirow[t]{2}{*}{$F_{[\mathrm{OIII}]}$} & \multirow[t]{2}{*}{ Class } & \multirow[t]{2}{*}{$z$} & \multirow{2}{*}{$\begin{array}{c}D_{L} \\
(\mathrm{Mpc})\end{array}$} & \multicolumn{2}{|c|}{$E(B-V)$} & \multirow[t]{2}{*}{ NED Name } \\
\hline & & & & & & & Gal. & AGN & \\
\hline PBC J0503.0+2300 & $\begin{array}{c}699 \pm 55 \\
{[2670 \pm 153]}\end{array}$ & $\begin{array}{c}113 \pm 23 \\
{[600 \pm 64]}\end{array}$ & $\begin{array}{c}93 \pm 7 \\
{[436 \pm 45]}\end{array}$ & Sy1.5 & 0.058 & 259.3 & 0.515 & 0.458 & 2MASX J05025822+2259520 \\
\hline PBC J0543.6-2738 & $\begin{array}{l}83.9 \pm 18.4 \\
{[88 \pm 14.9]}\end{array}$ & $\begin{array}{l}107 \pm 17 \\
{[120 \pm 9]}\end{array}$ & $\begin{array}{c}22.6 \pm 3.1 \\
{[25.4 \pm 3.2]}\end{array}$ & Sy1.2 & 0.009 & 38.8 & 0.029 & - & ESO 424- G 012 \\
\hline PBC J0814.4+0421 & $\begin{array}{c}394 \pm 35 \\
{[433 \pm 37]}\end{array}$ & $\begin{array}{c}43.9 \pm 7.7 \\
{[49.5 \pm 7.8]}\end{array}$ & $\begin{array}{c}31.9 \pm 2.3 \\
{[36.3 \pm 5.6]}\end{array}$ & NLS1 & 0.034 & 149.4 & 0.027 & 1.107 & CGCG 031-072 \\
\hline PBC J1345.4+4141 & $\begin{array}{c}27.3 \pm 1.5 \\
{[33.9 \pm 1.9]}\end{array}$ & $\begin{array}{c}0.7 \pm 0.2 \\
{[0.6 \pm 0.1]}\end{array}$ & $\begin{array}{c}3.7 \pm 1.8 \\
{[3.1 \pm 0.3]}\end{array}$ & Sy1.9 & 0.009 & 37.1 & 0.007 & 2.930 & NGC 5290 \\
\hline PBC J1439.0+1413 & $\begin{array}{c}16.5 \pm 3.1 \\
{[12.9 \pm 2.9]}\end{array}$ & - & - & Sy1.9 & 0.072 & 325.1 & 0.019 & - & 2MASX J14391186+1415215 \\
\hline PBC J1453.0+2553 & $\begin{array}{c}470 \pm 68 \\
{[1200 \pm 89]}\end{array}$ & $\begin{array}{c}111 \pm 22 \\
{[115 \pm 22.3]}\end{array}$ & $\begin{array}{c}19.7 \pm 3.3 \\
{[20.6 \pm 3.3]}\end{array}$ & Sy1 & 0.049 & 217.7 & 0.039 & 0.411 & 2MASX J14530794+2554327 \\
\hline PBC J1546.5+6931 & $\begin{array}{c}181 \pm 20 \\
{[244 \pm 23]}\end{array}$ & $\begin{array}{c}26 \pm 4.5 \\
{[29 \pm 4.8]}\end{array}$ & $\begin{array}{l}97.8 \pm 8.6 \\
{[113 \pm 16]}\end{array}$ & Sy1.9 & 0.037 & 162.9 & 0.041 & 1.069 & 2MASX J15462424+6929102 \\
\hline $\begin{array}{l}\text { Note: emission line fl} \\
\text { absorption } E(B-V)_{\mathrm{G}} \\
\text { The typical error on th } \\
\text { of } \pm 0.0003 \text { can be ass } \\
{ }^{*} \text { : blended with }[\mathrm{N} \text { II }]\end{array}$ & $\begin{array}{l}\text { xes are reporte } \\
\text { along the obj } \\
\text { e redshift meas } \\
\text { umed. } \\
\text { lines }\end{array}$ & $\begin{array}{l}\text { both as obse } \\
\text { t line of sigh } \\
\text { rement is } \pm 0\end{array}$ & $\begin{array}{l}\text { ed and (betw } \\
\text { (from Schleg } \\
01 \text { but for the }\end{array}$ & squar & $\begin{array}{l}\text { rackets) } \\
\text { ). Line } \\
\text { 6dFGS s }\end{array}$ & orrectec & or the & $\begin{array}{l}\text { ervenin } \\
10^{-15} \\
\text { uncert }\end{array}$ & $\begin{array}{l}\text { Galactic } \\
\mathrm{g} \mathrm{cm}^{-2} \mathrm{~s}^{-1} \\
\text { ty }\end{array}$ \\
\hline
\end{tabular}

\section{XMM analysis}

We report the results obtained from the X-ray data analysis of 3 out of 5 sources observed with $\mathrm{XMM} / \mathrm{Newton}$. We used data acquired with the pn X-ray CCD camera on the EPIC instrument onboard XMM. The other two objects were excluded for the following reasons: PBC J0041.6+2534 has a very low quality X-rays spectrum while the XMM data of PBC J0919.9+3712 have already been reported in the literature by Noguchi et al. (2009).

These data were processed using the Standard Analysis Software (SAS) version 9.0.0 employing the latest available calibration files and following usual procedures described in details in [12]. The spectral analysis has been performed using XSPEC v.12.6.0 and assuming initially a simple power law passing through Galactic ([6]) and intrinsic absorption; if this baseline model was not sufficient to fit the data, we then introduced extra spectral components as required, according to the F-test statistics.

\footnotetext{
${ }^{1}$ http://nedwww.ipac.caltech.edu/
} 
Table 2: Main results obtained from the analysis of the optical spectra of the 20 type 2 AGN of the present sample of Swift sources.

\begin{tabular}{|c|c|c|c|c|c|c|c|c|c|}
\hline \multirow[t]{2}{*}{ Object } & \multirow[t]{2}{*}{$F_{\mathrm{H}_{\alpha}}$} & \multirow{2}{*}{$F_{\mathrm{H}_{\beta}}$} & \multirow[t]{2}{*}{$F_{[\mathrm{OIII}]}$} & \multirow[t]{2}{*}{ Class } & \multirow[t]{2}{*}{$z$} & \multirow{2}{*}{$\begin{array}{c}\mathrm{D}_{L} \\
(\mathrm{Mpc})\end{array}$} & \multicolumn{2}{|c|}{$E(B-V)$} & \multirow[t]{2}{*}{ NED Name } \\
\hline & & & & & & & Gal. & AGN & \\
\hline PBC J0041.6+2534 & $\begin{array}{c}11.3 \pm 4.5 \\
{[12.1 \pm 3.8]}\end{array}$ & - & - & Sy2/LINER & 0.015 & 65.0 & 0.035 & - & NGC214 \\
\hline PBC J0100.6-4752 & $\begin{array}{c}37 \pm 4 \\
{[38 \pm 4]}\end{array}$ & $\begin{array}{l}10.8 \pm 2.9 \\
{[10.6 \pm 3]}\end{array}$ & $\begin{array}{c}101 \pm 6 \\
{[106 \pm 6]}\end{array}$ & Sy2 & 0.048 & 213.1 & 0.013 & 0.251 & ESO 195-IG 021 \\
\hline PBC J0122.3+5004 & $\begin{array}{c}139 \pm 23 \\
{[188 \pm 31]}\end{array}$ & $\begin{array}{c}34.1 \pm 5.7 \\
{[55 \pm 9]}\end{array}$ & $\begin{array}{c}169 \pm 28 \\
{[270 \pm 50]}\end{array}$ & Sy2 & 0.021 & 91.4 & 0.217 & 0.391 & MCG +08-03-018 \\
\hline PBC J0140.4-5320 & $\begin{array}{c}27.3 \pm 4.3 \\
{[31.6 \pm 4.8]}\end{array}$ & $\begin{array}{c}4.6 \pm 0.9 \\
{[5.4 \pm 0.9]}\end{array}$ & $\begin{array}{c}39.1 \pm 1.3 \\
{[42.7 \pm 1.4]}\end{array}$ & Sy2 & 0.072 & 325.1 & 0.029 & 0.725 & 2MASX J01402676-5319389 \\
\hline PBC J0248.9+2627 & $\begin{array}{c}47 \pm 4 \\
{[64.3 \pm 15.7]}\end{array}$ & $\begin{array}{c}4.2 \pm 0.5 \\
{[6.3 \pm 0.9]}\end{array}$ & $\begin{array}{c}21.6 \pm 1.5 \\
{[34.4 \pm 2.5]}\end{array}$ & Sy2 & 0.057 & 274.3 & 0.158 & 1.196 & 2MASX J02485937+2630391 \\
\hline PBC J0353.5+3713 & $\begin{array}{c}35.5 \pm 2.8 \\
{[36.7 \pm 2.8]}\end{array}$ & $\begin{array}{c}6 \pm 1 \\
{[5 \pm 0.9]}\end{array}$ & $\begin{array}{c}12.8 \pm 1.3 \\
{[13.2 \pm 1.3]}\end{array}$ & LINER & 0.019 & 82.6 & 0.536 & 0.938 & 2MASX J03534246+3714077 \\
\hline PBC J0356.9-4040 & $\begin{array}{l}75.6 \pm 11.8 \\
{[70.1 \pm 7.2]}\end{array}$ & $\begin{array}{c}21.7 \pm 5.6 \\
{[22.4 \pm 5.6]}\end{array}$ & $\begin{array}{c}167 \pm 9 \\
{[170 \pm 9]}\end{array}$ & Sy2 & 0.075 & 65.0 & 0.035 & 0.121 & 2MASX J03565655-4041453 \\
\hline PBC J0544.3+5905 & $\begin{array}{c}3.9 \pm 0.5 \\
{[6.4 \pm 0.8]}\end{array}$ & $\begin{array}{c}0.7 \pm 0.1 \\
{[1.4 \pm 0.2]}\end{array}$ & $\begin{array}{c}7.1 \pm 0.4 \\
{[15.9 \pm 0.8]}\end{array}$ & Sy2 & 0.068 & 306.2 & 0.274 & 0.484 & 2MASX J05442257+5907361 \\
\hline PBC J0623.8-3212 & $\begin{array}{c}97.9 \pm 17.4 \\
{[112 \pm 13.6]}\end{array}$ & - & $\begin{array}{c}783 \pm 40 \\
{[908 \pm 47]}\end{array}$ & Sy2 & 0.035 & 153.9 & 0.049 & - & ESO 426- G 002 \\
\hline PBC J0641.3+3251 & $\begin{array}{c}51.9 \pm 6.4 \\
{[69.4 \pm 10.4]}\end{array}$ & $\begin{array}{c}8.2 \pm 1.7 \\
{[13.3 \pm 2.1]}\end{array}$ & $\begin{array}{c}197 \pm 6.2 \\
{[311 \pm 20]}\end{array}$ & Sy2 & 0.049 & 217.7 & 0.153 & 0.611 & 2MASX J06411806+3249313 \\
\hline PBC J0759.9+2324 & $\begin{array}{c}8.9 \pm 1.1 \\
{[9.8 \pm 1.1]}\end{array}$ & $\begin{array}{c}0.7 \pm 0.1 \\
{[0.9 \pm 0.2]}\end{array}$ & $\begin{array}{c}3.6 \pm 0.5 \\
{[13.5 \pm 0.7]}\end{array}$ & Sy2 & 0.029 & 127 & 0.059 & 1.345 & CGCG 118-036 \\
\hline PBC J0919.9+3712 & $\begin{array}{c}4.3 \pm 0.4 \\
{[3.9 \pm 0.4]}\end{array}$ & $\begin{array}{c}0.5 \pm 0.1 \\
{[0.41 \pm 0.09]}\end{array}$ & $\begin{array}{l}4.1 \pm 0.3 \\
3.5 \pm 0.3\end{array}$ & Sy2 & 0.0075 & 32.3 & 0.012 & 1.118 & IC 2461 \\
\hline PBC J0954+3724 & $\begin{array}{c}8.6 \pm 0.5 \\
{[8.8 \pm 0.6]}\end{array}$ & $\begin{array}{l}0.8 \pm 0.03 \\
{[0.8 \pm 0.2]}\end{array}$ & $\begin{array}{c}3.6 \pm 0.6 \\
{[3.7 \pm 0.5]}\end{array}$ & Sy2 & 0.019 & 82.6 & 0.016 & - & IC 2515 \\
\hline PBC J1246.9+5433 & - & - & $\begin{array}{c}16.4 \pm 4.2 \\
{[17.8 \pm 4.4]}\end{array}$ & Sy2 & 0.017 & 73.8 & 0.017 & - & NGC 4686 \\
\hline PBC J1335.8+0301 & $\begin{array}{c}19.6 \pm 2.4 \\
{[20.7 \pm 1.9]}\end{array}$ & $\begin{array}{c}2.9 \pm 0.5 \\
{[3.2 \pm 0.5]}\end{array}$ & $\begin{array}{c}16.9 \pm 1.3 \\
{[17.9 \pm 1.1]}\end{array}$ & Sy2 & 0.0218 & 94.9 & 0.024 & 0.830 & NGC 5231 \\
\hline PBC J1344.2+1934 & $\begin{array}{c}16.6 \pm 1.8 \\
{[17.2 \pm 1.8]}\end{array}$ & - & $\begin{array}{c}6.6 \pm 1.1 \\
{[6.9 \pm 1.2]}\end{array}$ & Sy2/LINER & 0.027 & 118 & 0.027 & - & CGCG 102-048 \\
\hline PBC J1506.6+0349 & $\begin{array}{c}17.2 \pm 1.1 \\
{[19.4 \pm 2.6]}\end{array}$ & $\begin{array}{c}2.5 \pm 0.6 \\
{[2.7 \pm 0.7]}\end{array}$ & $\begin{array}{c}20.8 \pm 0.8 \\
{[23.7 \pm 1.4]}\end{array}$ & Sy2 & 0.038 & 167.5 & 0.049 & 0.908 & 2MASX J15064412+0351444 \\
\hline PBC J2148.2-3455 & $\begin{array}{c}6460 \pm 582 \\
{[6900 \pm 895]}\end{array}$ & $\begin{array}{c}857 \pm 83 \\
{[947 \pm 117]}\end{array}$ & $\begin{array}{c}4970 \pm 347 \\
{[5440 \pm 347]}\end{array}$ & Sy2 & 0.0161 & 70.7 & 0.029 & - & NGC 7130 \\
\hline PBC J2333.9-2343 & - & $\begin{array}{c}3.2 \pm 1.2 \\
{[3.5 \pm 1.2]}\end{array}$ & $\begin{array}{c}14.7 \pm 2.8 \\
{[16.3 \pm 2.8]}\end{array}$ & Sy2 & 0.0475 & 210.8 & 0.029 & - & PKS 2331-240 \\
\hline PBC J2341.9+3036 & $\begin{array}{c}9.7 \pm 4.6 \\
{[14.2 \pm 5.3]}\end{array}$ & - & $\begin{array}{c}8.3 \pm 2.1 \\
{[15.6 \pm 2.9]}\end{array}$ & Sy2 & 0.017 & 73.8 & 0.102 & - & UGC 12741 \\
\hline $\begin{array}{l}\text { Note: emission line } \mathrm{f} \\
\text { absorption } E(B-V) \\
\text { The typical error on } t \\
\text { of } \pm 0.0003 \text { can be as }\end{array}$ & $\begin{array}{l}\text { uxes are reporte } \\
\text { al along the obje } \\
\text { ee redshift meas } \\
\text { umed. }\end{array}$ & ement is \pm 0 . & $\begin{array}{l}\mathrm{d} \text { and (betwee } \\
\text { rom Schlegel } \\
1 \text { but for the }\end{array}$ & $\begin{array}{l}\text { quare brack } \\
\text { al. 1998). Li } \\
\text { sS and 6dFC }\end{array}$ & $\begin{array}{l}\text { correct } \\
\text { fluxes a } \\
\text { spectra, }\end{array}$ & or the & $\begin{array}{l}\text { ervening } \\
10^{-15} \mathrm{er} \\
\text { uncertair }\end{array}$ & $\begin{array}{l}\text { Galact } \\
\mathrm{g} \mathrm{cm}^{-2} \\
\text { nty }\end{array}$ & \\
\hline
\end{tabular}

The results of this analysis are reported in Table 4, where we list the Galactic absorption, the column density in excess to the Galactic value, the power law photon index, the reduced $\chi^{2}$ of the best-fit model, the 2-10 keV flux and 20-100 keV fluxes; extra spectral parameters if required are discussed in the text. Quoted errors correspond to $90 \%$ confidence level for a single parameter of interest $\left(\Delta \chi^{2}=2.71\right)$. 
Table 3: Main results concerning PBC J0826.3-7033 identified as a cataclismic variable.

\begin{tabular}{|c|c|c|c|c|c|c|c|c|c|c|}
\hline \multirow[t]{2}{*}{ Object } & \multicolumn{2}{|c|}{$\mathrm{H}_{\alpha}$} & \multicolumn{2}{|c|}{$\mathrm{H}_{\beta}$} & \multicolumn{2}{|c|}{ He II $\lambda 4686$} & \multirow{2}{*}{$\begin{array}{c}\text { Optical } \\
\text { mag. }\end{array}$} & \multirow{2}{*}{$\begin{array}{c}A_{V} \\
(\mathrm{mag})\end{array}$} & \multirow{2}{*}{$\begin{array}{c}d \\
(\mathrm{pc})\end{array}$} & \multirow[t]{2}{*}{$L_{\mathrm{X}}$} \\
\hline & EW & Flux & EW & Flux & EW & Flux & & & & \\
\hline PBC J0826.3-7033 & $38.9 \pm 1.8$ & $66 \pm 3$ & $33.6 \pm 1.5$ & $44 \pm 2$ & $5.7 \pm 0.9$ & $7.4 \pm 1.1$ & $13.8(R)$ & 0 & 90 & $\begin{array}{r}0.002(2-10) \\
0.007(20-100)\end{array}$ \\
\hline
\end{tabular}

\subsection{PBC J1246.9+5433}

The best fit model $\left(w a_{g a l} *(b b+w a *(p o+g a+g a))\right)$ to this source requires an extra black body component with a $k T=0.28_{-0.03}^{+0.04} \mathrm{keV}$ and two narrow lines at $E=6.29_{-0.03}^{+0.03} \mathrm{keV}$ and $6.79_{-0.10}^{+0.11}$ $\mathrm{keV}$ with an EW of $600_{-174}^{+182} \mathrm{eV}$ and $378_{-167}^{+168} \mathrm{eV}$ respectively. The other parameters of the baseline model are reported in Table 4. The presence of strong excess emission below $1 \mathrm{keV}$ and of two prominent lines around 6.3 and $6.8 \mathrm{keV}$, together with the extremely flat power law strongly point to a highly absorbed AGN. Indeed, PBC J1246.9+5433 shows an intrinsic absorption of $23.6_{-9.6}^{+8.0}$ $\times 10^{22} \mathrm{~cm}^{-2}$. This object has been classified as a Seyfert 2 from our optical spectroscopic analysis and its X-ray spectrum is fully compatible with its optical type.

\subsection{PBC J1335.8+0301}

The best fit for the X-ray spectrum of this source is obtainedusing a simpler model $\left(w a_{g a l} *\left(p o+w a^{*} p o\right)\right)$ than that of PBC J1246.9+5433. With respect to our baseline model, we only found an extra power law component having the same photon index of the primary absorbed power law but passing only through the Galactic column density. The photon index is flat $(\Gamma=$ $1.58_{-0.04}^{+0.06}$ ) while the intrinsic column density is $2.3^{ \pm 0.1} \times 10^{22} \mathrm{~cm}^{-2}$. Also PBC J1335.8+0301 is classified as a Seyfert 2 galaxy in optical, although the X-ray data suggest that this is a mildly absorbed type 2 AGN.

\subsection{PBC J2341.9+3036}

Also in this case the best fit model shows some extra features $\left(w a_{g a l} *(p o+w a *(p o+g a))\right)$. It requires a second power law component having the same photon index of the primary absorbed power law and passing only through the Galactic column density, as well as a narrow emission line at $E=6.25_{-0.06}^{+0.05}$ with an $\mathrm{EW}=365_{-147}^{+176} \mathrm{eV}$. The primary continuum has a steep photon index $(\Gamma=$ $2.02_{-0.15}^{+0.16}$ ) and a column density of $56.5_{-10.4}^{+15.5} \times 10^{22} \mathrm{~cm}^{-2}$, which makes PBC J2341.9+3036 one of the most absorbed sources in our sample, in agreement with its optical classification as a Seyfert 2 galaxy.

\section{Conclusions}

With this work we have been able to either give or confirm or correct the optical classification of 28 Swift sources belonging to the Palermo 39 months Swift catalogue (see also [12] for details). This was achieved through a multisite observational campaign in Europe, South Africa, Central and South America. 
Table 4: Main results obtained from the analysis of the X-ray spectra of 3 out of 5 objects observed with $\mathrm{XMM} / \mathrm{Newton}$ in the present sample.

\begin{tabular}{ccccccc}
\hline \hline Source & $\begin{array}{c}\mathrm{N}_{\mathrm{Hgal}} \\
\times 10^{22} \mathrm{~cm}^{-2}\end{array}$ & $\Gamma$ & $\begin{array}{c}\mathrm{N}_{H} \\
\times 10^{22} \mathrm{~cm}^{-2}\end{array}$ & $\begin{array}{c}\chi^{2} / v \\
\times 10^{-11} \mathrm{erg} \mathrm{s}^{-1} \mathrm{~cm}^{-2}\end{array}$ & $\begin{array}{c}\mathrm{F}_{(20-100) \mathrm{kVV}} \\
\times 10^{-11} \mathrm{erg} \mathrm{s}^{-1} \mathrm{~cm}^{-2}\end{array}$ \\
\hline PBC J1246.9+5433 & 0.014 & $0.88_{-0.12}^{+0.13}$ & $23.6_{-9.6}^{+8.0}$ & $15 / 22$ & 0.09 & 1.5 \\
PBC J1335.8+030 & 0.019 & $1.58_{-0.04}^{+0.06}$ & $2.3_{-0.1}^{+0.1}$ & $313.3 / 299$ & 0.64 & 1.1 \\
PBC J2341.9+3036 & 0.058 & $2.02_{-0.15}^{+0.16}$ & $56.5_{-10.4}^{+15.5}$ & $25 / 28$ & 0.11 & 1.0 \\
\hline \hline
\end{tabular}

We found that our sample is composed of 27 AGNs (7 of Type 1 and 20 of Type 2), with redshifts between 0.009 and 0.075 , and $1 \mathrm{CV}$. Among these sources we found some peculiar objects, such as 3 likely LINERs and 1 narrow line seyfert 1.

The X-ray spectral analysis of 3 out of 5 sources observed with XMM-Newton shows a complex best-fit model with an absorbed power law component as a primary emission model; all 3 require an extra component at low energies to fit an emission excess below few $\mathrm{keV}$, while only two display iron line emission features.

This work shows the importance of optical spectroscopic follow up observations for sources discovered by hard X-ray surveys and either unclassified or poorly studied. By increasing the number of the identifications in hard X-ray catalogues, it is possible to perform more reliable statistical studies as multiwavelength characterization of the sources, thus allowing a better understanding of the physical processes that drive the powerful AGN detected.

\section{References}

[1] A.D. Barthelmy, L.M. Barbier, J.R. Cummings, et al., 2005, 120, 143

[2] D.N. Burrows, J.E. Hill, J.A. Nousek, et al. 2005, Sp.Sci.Rev. 120, 165

[3] A. Comastri, 2004, Supermassive Black Holes in the Distant Universe, ed. A.L. Barger (Dordrecht, The Netherlands: Kluwer Academic Publishers), 308, 245

[4] G. Cusumano, V. La Paola, A. Segreto, et al. 2010a, A\&A, 510, 48

[5] G. Cusumano, V. La Parola, A. Segreto, et al. 2010b, A\&A, 524, 64

[6] J.M. Dickey \& F.J. Lockman, 1990, A\&A, 28, 215

[7] N. Gehrels, G. Chincarini, P. Giommi, et al. 2004, ApJ, 611, 1005

[8] N. Masetti, P. Parisi, E. Palazzi, et al. 2010, A\&A 519, A96

[9] D.G Monet, S.E. Levine, B. Canzian, et al. 2003, AJ, 125, 984

[10] K. Noguchi, Y. Terashima, H. Awaki, 2009, ApJ, 705, 454

[11] P. Parisi, N. Masetti, E. Jiménez-Bailón, et al. 2009, A\&A, 507, 1345

[12] P. Parisi, N. Masetti, E. Jiménez-Bailón, et al. 2011, in preparation

[13] P.W.A. Roming, S.D. Hunsberger, K.O. Mason, et al. 2004, Proceedings of the SPIE, 5165, 262

[14] J. Tueller, W.H. Baumgartner, C.B Markwardt, et al. 2010, ApJS 186, 378 